\title{
ON THE NUMBER OF SINGULARITIES IN MEROMORPHIC FOLIATIONS ON COMPACT COMPLEX SURFACES
}

\author{
EDOARDO BALLICO
}

\begin{abstract}
Here we study meromorphic foliations with singularities on a smooth compact complex surface. Aim: study the locations of the singularities, using vector bundle techniques and techniques introduced in the cohomological study of projective geometry.
\end{abstract}

Roughly speaking, a meromorphic foliation with singularities on a complex connected manifold $S$ is described by a subsheaf $F$ of the tangent bundle $T S$ with $F$ closed under Lie brackets (see for instance [G1, G2, G3, G4, G5, $\mathrm{GK}, \mathrm{GM}]$ or, for the case $\operatorname{dim}(S)=2$, here in 1.8.1). The singularities of the foliations are the points of $S$ at which $F$ is not a subbundle of $T S$; with this definition, the singularities may occur only in codimension at least 2 (but a meromorphic foliation without singularities is not a holomorphic foliation in the classical sense). Note that any rank one subsheaf of $T S$ is involutive and it is contained in a line bundle contained in $T S$ (in general not as subbundle). Thus a rank one meromorphic foliation $G$ with singularities on $S$ is given by a line bundle $M$ on $S$ and a nonzero map $M \rightarrow T S$ (which induces an inclusion as sheaves of $M$ in $T S$ ) (up to a scalar factor, of course); one imposes that $(s)_{0}$ has no hypersurface as component (i.e. that $M$ is a saturated subsheaf of $T S$ ); equivalently, $G$ is given by $M$ and $s \in H^{0}\left(S, T S \otimes M^{*}\right)$ with $s \neq 0$ (up to a scalar factor); the singularities of $G$ are the zero locus $(s)_{0}$ of $s$. Such a foliation is called also a foliation by curves. Thus, for fixed $M \in \operatorname{Pic}(S)$, we are looking at a linear problem. Many papers are concerned with this case (see e.g. $[G 2, G 3, G 4, G K]$ ) from different points of view and with several different kinds of problems in mind. When $\operatorname{dim}(S)=2$ (as always in this paper) every foliation is a foliation by curves. For the case $\operatorname{dim}(S)=2$, relevant references are (among others) [G2 and G3]. From now on we assume $\operatorname{dim}(S)=2$. It happens very seldom that $S$ has a meromorphic foliation without singularities; by definition this happens if and only if $T S$ is an extension of two line bundles. Here we are interested essentially on the possible location (for various $M \in$ $\operatorname{Pic}(S))$ of the singularities associated to some $s \in H^{0}\left(S, T S \otimes M^{*}\right)$; very often we will be interested in a general such $s$. The main problems considered here are:

(a) given a zero dimensional scheme $Z \subset S$, find if there is

$$
s \in H^{0}\left(S, T S \otimes M^{*}\right), \quad s \neq 0,
$$

with $Z \subseteq(s)_{0}$;

Received by the editors November 29, 1990 and, in revised form, November 5, 1992.

1980 Mathematics Subject Classification (1985 Revision). Primary 58F18, 14 J26. 
(b) find for what integer $t$, we may find $s \in H^{0}\left(S, T S \otimes M^{*}\right), s \neq 0$, with $(s)_{0}$ containing $t$ general points of $S$.

Several of the definitions and techniques used in this paper have nothing to do with foliations; they depend only on the choice of a rank two vector bundle $E$. Such definitions/results are contained in $\S \S 1$ and 2 . We stress that some of the definitions seem to be very useful for the study of the geometry of the pair $(S, E)$. The fact is that for foliations $E \cong T S \otimes M^{*}$ enters only when we consider the examples. We use of course the classification of surfaces. In (2.4) we consider the case of a smooth surface $S \subset \mathbf{P}^{3}$; in 2.4 .1 we compute (for large $x$ ) the maximal integer $t$ such that we have $h^{0}\left(S, T S \otimes \mathscr{O}_{S}(x) \otimes \mathscr{I}_{W}\right)=$ $h^{0}\left(S, T S \otimes \mathcal{O}_{S}(x)\right)-2 t$ for a general $W \subset S$ with $\operatorname{card}(W)=2 t$. In (3.1) and (3.3) we consider respectively the case of $\mathbf{P}^{2}$ and of a rational ruled surface. In (3.5) we say a few words for the case of a surface of general type. Section 4 is propaganda. It shows with an example (the blowing-up, $F_{1}$, of $\mathbf{P}^{2}$ at one point: see 4.1) how easy it is to use (even for a user, not a specialist) the socalled Horace method (due to A. Hirschowitz) to handle problems (a) and (b) (or related problems) and to construct examples related to these problems. In $\S 5$ we consider briefly the case of a nonalgebraic surface. In $\S 6$ we consider the case of foliations by curves with logarithmic singularities along a normal crossing divisor; see $\S 6$ for the relevant definitions (e.g. the notion of vector field with logarithmic poles along a normal crossing divisor). Theorem 6.1 considers the case of logarithmic vector fields over $\mathbf{P}^{2}$. Here we stress only that 6.1 is another application of the Horace method.

We work over an algebraically closed field of characteristic zero (leaving the positive characteristic case to Remark 2.6). Of course, when we speak about holomorphic or meromorphic objects, we are in the case of complex numbers and we may consider also nonalgebraic objects (essentially nonalgebraic complex compact surfaces as in $\S 5$ ).

We now fix a few notations. Fix a smooth, compact complex surface $S$. Set $\mathscr{O}:=\mathscr{O}_{S}$. For any subscheme $U$ of a scheme $V$, let $\mathscr{I}_{U, V}$ be its defining ideal sheaf in $V$; if $V=S$, we will write $\mathscr{I}_{U}$ instead of $\mathscr{I}_{U, V}$. For the cohomology groups (resp. their dimensions) of a sheaf $G$ on $S$, we will write $H^{i}(G)$ (resp. $\left.h^{i}(G)\right)$ instead of $H^{i}(S, G)$ (resp. $h^{i}(S, G)$ ). Fix a rank two vector bundle $T$ on $S$, line bundles $M, R$ and $A \subset S, A$ a zero-dimensional scheme. We assume given (and fixed) the following exact sequence:

$$
0 \rightarrow M \rightarrow T \rightarrow R \otimes \mathscr{J}_{A} \rightarrow 0 .
$$

For its relation to the title of this paper, see 1.8. We will always use $M, T, R$ and $A$ with this meaning and fitting in (1). Note that (1) implies that $\operatorname{det}(T) \cong$ $M \otimes R, A$ is locally defined by two equations and $c_{2}(T)=\operatorname{length}(A)+M \cdot R$. To have an exact sequence like (1) for some zero-dimensional $A$ is equivalent to give an homomorphism, $y$, from $M$ to $T$ which drops rank only in codimension two; then $A$ is the scheme $(y)_{0}$ where $y$ drops rank, i.e., $\mathscr{I}_{A}$ is defined in the following way; $y$ corresponds to $y^{\prime} \in H^{0}(T(-M))$; taking duals, $y^{\prime}$ induces $y^{\prime \prime}:(T(-M))^{*} \rightarrow \mathscr{O} ; \mathscr{I}_{A}$ is by definition $\operatorname{Im}\left(y^{\prime \prime}\right)$. Set $a(1):=h^{0}(M)$ and let $a(2)$ be the dimension of the image of $H^{0}(T)$ into $H^{0}\left(R \otimes \mathscr{J}_{A}\right)$ for the 
map induced by (1); set $b(M, T):=\min (a(1), a(2))$; set $b^{\prime}(M, t):=b(M, T)$ if $a(1) \neq a(2)$, and $b^{\prime}(M, T):=b(M, T)-1$ if $a(1)=a(2)$.

Proposition 1.1. If $x \leq b^{\prime}(M, T)$, for a general subset $B \subset S$ with $\operatorname{card}(B)=x$ there is $s \in H^{0}(T)$ with $\operatorname{dim}\left((s)_{0}\right)=0,(s)_{0}$ reduced and with $B \subseteq(s)_{0}$. Furthermore, $h^{0}\left(T \otimes \mathscr{I}_{B}\right)=h^{0}(T)-2 x$.

Proof. Tensor (1) with $\mathscr{I}_{B}$. By the generality of $B$, we have $h^{0}\left(M \otimes \mathscr{I}_{B}\right)=$ $h^{0}(M)-x$ and $\operatorname{dim}\left(\operatorname{Im}\left(H^{0}\left(T \otimes \mathscr{I}_{B}\right) \rightarrow H^{0}\left(R \otimes \mathscr{I}_{A} \otimes \mathscr{I}_{B}\right)\right)\right)=a(2)-x$, hence $h^{0}\left(T \otimes \mathscr{I}_{B}\right)=h^{0}(T)-2 x>0$ (by the definition of $b^{\prime}(M, T)$ ). A general $w \in H^{0}(T)$ has $(w)_{0}$ reduced and of dimension 0 (Bertini theorem); since $h^{0}\left(T \otimes \mathscr{J}_{B}\right)=h^{0}(T)-2 x$ and the set of such general $B$ has dimension $2 x$, a standard justification of a dimensional count gives that for general $B$ a general $s \in H^{0}\left(T \otimes \mathscr{J}_{B}\right)$ has $\operatorname{dim}\left((s)_{0}\right)=0$ and $(s)_{0}$ is reduced.

A double point of $S$ is a length-two subscheme of $S$ (hence it has dimension zero and it is isomorphic to $\left.\operatorname{Spec}\left(\mathbf{C}[\varepsilon] /\left(\varepsilon^{2}\right)\right)\right)$.

Lemma 1.2. Fix $L \in \operatorname{Pic}(S)$ and a vector space $W \subseteq H^{0}(L)$. Fix an integer $y$ with $2 y<\operatorname{dim}(W)$. Then for a general $B \subset S$ with $\operatorname{card}(B)=y$, there is a scheme $Z \subset S$ with $Z_{\mathrm{red}}=B$, length $(Z)=2 y$ and $Z$ a union of $y$ double points, and such that the vector space $\{w \in W: w$ vanishes on $Z\}$ has codimension $2 y$ in $W$.

Proof. First assume $y=1$. Let $|W|$ be the linear system associated to $W$. Since by assumption $\operatorname{dim}(W) \geq 2,|W|=J+|I|$ with $J$ fixed part (maybe $J=\varnothing$ ) and $|I|$ mobile part; let $J^{\prime}$ be the base locus of $|W|$ (hence $J^{\prime} \backslash J$ is finite). A point of $S \backslash J^{\prime}$ imposes one condition on $W$. Since we are in characteristic zero a general $C \in|I|$ is reduced; fix $a$ such that $C$ and $P \in C_{\text {reg }}$ with $P \notin J^{\prime}$. Since $C$ is smooth at $P$, no double point $v$ with support $P$ and not tangent to $C$ at $P$ is contained in $C$. Hence $v$ imposes one more condition to $W$ than $P$, proving the case $y=1$. If $y \geq 2$, take by induction $B^{\prime}$ and $Z^{\prime}$ working for the case $y^{\prime}:=y-1$, and apply the case $y=1$ to $W^{\prime}:=\left\{w \in W: w\right.$ vanishes on $\left.Z^{\prime}\right\}$.

Using 1.2 and the first part of the proof of 1.1 we obtain the following result.

Proposition 1.3. Fix integers $x \geq 0, y \geq 0$ with $x+2 y \leq b^{\prime}$. Then for general subsets $B, B^{\prime}$ with $\operatorname{card}(B)=x, \operatorname{card}\left(B^{\prime}\right)=y$, and a general scheme $Z^{\prime} a$ union of $y$ double points with support $B^{\prime}, H^{0}\left(T \otimes \mathscr{I}_{B \cup Z^{\prime}}\right)$ has codimension $x+2 y$ in $H^{0}(T)$.

For low $y$ (and often for many $y$ ) it is easy to prove that, with the notations of 1.3, a general $s \in H^{0}\left(T \otimes \mathscr{F}_{B \cup Z^{\prime}}\right)$ has $\operatorname{dim}\left((s)_{0}\right)=0$ and $(s)_{0}$ is, as a scheme, the disjoint union of $Z^{\prime}$ and a reduced scheme (formed by $c_{2}(T)-2 y$ points).

Definition 1.4. The vector bundle $T$ is called $h^{0}$-semistable (resp. $h^{0}$-stable) if for every exact sequence (1) we have $a(1)<a(2)$ (resp. $a(1) \leq a(2)) . T$ is called $h^{0}$-unstable if it is not $h^{0}$-semistable; if in (1) we have $a(1)>a(2)$, (1) will be called a $h^{0}$-destabilizing sequence for $T$.

This definition (for curves) was given by Perrin [Pe, Definition 3.11] in his study of the normal bundle of a curve in $\mathbf{P}^{3}$. This definition works for rank two vector bundles on any variety. It seems quite useful. In this paper we need exactly Definition 1.4 , e.g. to state concisely the next result which settles 
the case $a(1)>a(2)$; vice versa, 1.5 shows that 1.4 is very reasonable in this context.

Proposition 1.5. Assume $a(1)>a(2)$ i.e. that (1) is a $h^{0}$-destabilizing sequence for $T$. Fix an integer $x$ with $a(2) \leq x<a(1)$ and let $B$ be a general subset of $S$ with $\operatorname{card}(B)=x$. Then $h^{0}\left(T \otimes \mathscr{I}_{B}\right)=h^{0}(T)-a(2)-x$. However every $s \in H^{0}\left(T \otimes \mathscr{J}_{B}\right)$ vanishes on a curve belonging to the linear system associated to $M$.

Proof. Take a general $B$ and write $B=B^{\prime} \cup B^{\prime \prime}$ with $\operatorname{card}\left(B^{\prime}\right)=a(2)$, $\operatorname{card}\left(B^{\prime \prime}\right)=x-a(2)$. Tensoring (1) with $\mathscr{I}_{B}$ and applying 1.1 to $B^{\prime}$ we see easily that $h^{0}\left(T \otimes \mathscr{I}_{B}\right)=h^{0}(T)-a(2)-x$. Fix $s \in H^{0}\left(T \otimes \mathscr{I}_{B}\right)$. By the choice of $B=B^{\prime} \cup B^{\prime \prime}$ we see that the image of $s$ in $h^{0}\left(R \otimes \mathscr{I}_{A}\right)$ vanishes, i.e. $s$ comes from a section of $M$ (which by definition of line bundle vanish on a suitable curve).

We note explicitly that for a spanned vector bundle $E$ on $S$ a general $s \in$ $H^{0}(E)$ may not vanish on a general set $B \subset S$ with $\operatorname{card}(B)=c_{2}(E)$, even if $h^{0}(E)>2 c_{2}(E)$ and hence for any such $B$ there is $y \in H^{0}(E)$ with $B \subseteq(y)_{0}$; the reason is that, for general $B$, it may happen that for any such $y$ we have $\operatorname{dim}\left((y)_{0}\right)=1$ (example: $\left.S=\mathbf{P}^{2}, E=\mathscr{O}(3) \oplus \mathscr{O}(1)\right)$; this is an explanation of a geometric consequence of $h^{0}$-instability.

If $a(1)<a(2)$, in the range $x>b(M, T)$ nothing can be said (a priori), since the picture depends very much on $T$. For instance, if $T=M \oplus R$, we can exchange the role of $M$ and $R$ and find a $h^{0}$-destabilizing sequence for $T$; thus in this case 1.5 applies. But there are examples (see [B, Remark 3.1], for the case $T=T \mathbf{P}^{2}(t)$ with $t$ any integer) for which 1.1 holds for every integer $x$ with $2 x<h^{0}(T)$ (i.e. the best possible result holds).

In the next section we will need the following trivial remark.

Remark 1.6. Fix (1) and let $H \in \operatorname{Pic}(S)$ be an ample line bundle. Twist (1) by $n H$ and call $a(i, n), i=1,2$, the corresponding integers (hence $\left.a(1, n):=h^{0}(M(n H))\right)$. By Riemann-Roch and Serre vanishing, for high $n$ each $a(i, n)$ increases quadratically as $\left(H^{2} / 2\right) n^{2}, h^{0}(T(n H))$ increases quadratically as $\left(H^{2}\right) n^{2}$, while $|a(1, n)-a(2, n)|$ increases at most linearly with $n$.

(1.7) We note explicitly that in the proofs of 1.1 and 1.3 (and in most of their applications) we use the generality of the subsets of $S$ with suitable cardinality only to say that their points impose the maximal possible number of conditions to certain explicitly given linear systems. Hence in explicit cases one can (sometimes) find example; anyhow, the rank two problem is reduced to two rank one problems. The Horace method (see $\S 4$ ) is often useful for the construction of these examples.

(1.8) Here we recall the definition of meromorphic foliation with singularities on $S$ and its link with the exact sequence (1).

Definition 1.8.1. Fix a line bundle, $L$, on $S$. A meromorphic foliation on $S$ with $L$ as pole divisor is a section $s$ of $T S \otimes L$ (up to a multiplicative nonzero constant) whose zero locus, $A$, is either empty or supported by finitely many points.

Note that if we set $M:=L^{*}, R:=\operatorname{det}(T S) \otimes L$ and $T:=T S$, a meromorphic foliation with $L$ as pole divisor and "singular locus" $A$ is just the datum 
of the exact sequence (1). Note that here the integrability condition "closed under Lie bracket" is automatically satisfied because $\operatorname{rank}(M)=1$.

2

This section is both a continuation of $\S 1$ (hence with definitions and results working for any rank two vector bundle) and the beginning of the application of these definitions and results to the case of the tangent bundle $T S$ of a smooth surface. We fix a smooth complete connected surface (or a compact complex smooth surface) and use the notations introduced in the previous section. We fix a rank two vector bundle $E$ on $S$. In many examples we will have $E \cong T S(L)$ with $L \in \operatorname{Pic}(S)$.

The following general definition seems to be very useful.

Definition 2.1. Let $d(E)$ be the maximal integer $t$ such that for a general subset $W \subset S$ with $\operatorname{card}(W)=t$, we have $h^{0}\left(E \otimes \mathscr{J}_{W}\right)=h^{0}(E)-2 t$. Set $\tau(E):=h^{0}(E)-2 d(E)-1$.

(2.2) Here we want to show that $h^{0}$-instability is a rather common phenomenon for the vector bundles which are relevant to meromorphic foliations (see also (2.3.2)). Fix $L \in \operatorname{Pic}(S)$. Fix a general $W \subset S$ with $\operatorname{card}(W)=$ $d(T S(L))$. Assume $h^{0}(T S(L))>0$ and $\tau(T S(L))>0$. Let $\pi: S^{\prime} \rightarrow S$ be the blowing-up of $S$ at $W$. The vector fields on $S^{\prime}$ corresponds bijectively to the vector fields on $S$ vanishing at every point of $W$ (see e.g. [G3, $\S 4])$. Hence by the definition of $d(E)$ we see that for a general $P \in S^{\prime}$ we have $h^{0}\left(T S^{\prime}\left(\pi^{*}(L)\right) \otimes \mathscr{I}_{\{P\}}\right)=h^{0}\left(T S^{\prime}\left(\pi^{*}(L)\right)\right)-1$. Hence the subsheaf, $J$, of $T S^{\prime}\left(\pi^{*}(L)\right)$ has rank one, i.e. $J^{* *} \in \operatorname{Pic}\left(S^{\prime}\right)$; let $M$ be the saturation of $J$ in $T S^{\prime}\left(\pi^{*}(L)\right)$, i.e. let $M \subset T S^{\prime}\left(\pi^{*}(L)\right)$ with $J \subseteq M, \operatorname{rank}(M)=1$, and $T S^{\prime}\left(\pi^{*}(L)\right) / M$ torsion-free; we have $M \in \operatorname{Pic}\left(S^{\prime}\right)$. Hence the inclusion of $M$ in $T S^{\prime}\left(\pi^{*}(L)\right)$ induces an exact sequence like (1). By the definition of $J$, this is an $h^{0}$-destabilizing sequence for $T S^{\prime}\left(\pi^{*}(L)\right)$.

(2.3) Now we relate the two concepts, $h^{0}$-stability and $d(E)$, for a rank two vector bundle $E$ on a projective (or complex and compact) smooth surface; we assume $h^{0}(E)>0$ (otherwise everything is meaningless) and set $d:=d(E)$.

(2.3.1) First assume $2 d=h^{0}(E)$. The relevant example (see (3.1)) is the case $(S, E)=\left(\mathbf{P}^{2}, T \mathbf{P}^{2}(t)\right)$ for every integer $t$ with $h^{0}\left(T \mathbf{P}^{2}(t)\right)$ even, i.e. by the Euler sequence for every even $t$. A trivial example: if $E \cong I \oplus I^{\prime}$ with $I$ and $I^{\prime}$ line bundles, the assumption of $(2.3 .1)$ means $h^{0}(I)=h^{0}\left(I^{\prime}\right)$. By 1.1 the assumption of (2.3.1) implies that $E$ is $h^{0}$-semistable. Fix a general $B \subset S$ with $\operatorname{card}(B)=d-1$. Let $J$ (or $J_{B}$ if you prefer) the double dual of the image of the natural evaluation map from $H^{0}\left(E \otimes \mathscr{I}_{B}\right) \otimes \mathcal{O}$ to $E$. By definition of $d$, we have $\operatorname{rank}(J)=2, h^{0}(J)=2$, and $J$ is generically spanned by its global sections.

(2.3.2) Now assume $2 d \leq h^{0}(E)-1$ (by far the most common case). Fix a general $B \subset S$ with $\operatorname{card}(B)=d$. Let $J \in \operatorname{Pic}(S)$ (or $J_{B}$ if you prefer) the saturation of the double dual of the image of the natural evaluation map from $H^{0}\left(E \otimes \mathscr{I}_{B}\right) \otimes \mathscr{O}$ to $E$ (note that by the definition of $d$ this image has (generically) rank one). By definition of saturation we have an exact sequence of type (1),

$$
0 \rightarrow J \rightarrow E \rightarrow R \otimes \mathscr{I}_{A} \rightarrow 0
$$


with $R \cong \operatorname{det}(E) \otimes J^{*}$ and $\operatorname{dim}(A)=0$. Note that $h^{0}(J) \geq d$ by the definition of $J$. If $2 d=h^{0}(E)-1$, note that $a(1) \neq a(2)$. By 1.1 we have $d=$ $h^{0}(E)-h^{0}(J)$. Given $E$ (hence $h^{0}(E)$ ) if $\left\{h^{0}(I): I \in \operatorname{Pic}(S)\right\}$ is a small subset of the natural numbers (as in several very interesting cases) this gives a very strong restriction on $d$.

(2.4) Now we apply the previous subsection (2.3) to analyze the important case of a smooth surface $S \subset \mathbf{P}^{3}$ (leaving to the interested reader to make the change needed in the case of more general complete intersection smooth surfaces in $\left.\mathbf{P}^{n}\right)$. Fix a smooth $S \subset \mathbf{P}^{3}$; set $s:=\operatorname{deg}(S)>1$. Write $\mathbf{P}$ instead of $\mathbf{P}^{3}$. Here $\Omega_{V}$ means $\Omega_{V}^{1}$ (the cotangent bundle) and we drop the subscript $V$ if $V=S$. Fix an integer $x$. We want to show how to compute $d(T S(x))$. It is known that $\Omega_{\mathbf{P}}(2)$ is spanned by its global sections. Consider a twist of the conormal bundle exact sequence of $S$,

$$
0 \rightarrow \mathscr{O}(t-s) \rightarrow \Omega_{\mathbf{P}}(t) \mid S \rightarrow \Omega(t) \rightarrow 0 .
$$

Note that $\Omega \cong T S \otimes K \cong T S(d-4)$. By (3) $\Omega(2)$ is spanned and (by Bertini theorem) we have an exact sequence

$$
0 \rightarrow \mathscr{O}(x+2-s) \rightarrow T(x) \rightarrow \mathscr{I}_{A}(x+2) \rightarrow 0
$$

with $A \subset S, A$ reduced, and $\operatorname{card}(A)=c_{2}(\Omega(2))=s(s-2)$ (use (3) and the Euler sequence for $\Omega_{\mathbf{P}}$. By (4) and 1.1 we have $d(T(x)) \geq h^{0}(\mathscr{O}(x+2-s)$ ) (at least for large $x$ (with respect to $s$; how large is a tedious but straightforward explicit calculation). We want to prove the following result.

Proposition 2.4.1. We have $d(T(x))=h^{0}(\mathscr{O}(x+2-s)$ ) for large $x$.

Note that by 2.4.1 $d(T(x))$ is explicitly known and computable (if necessary).

Proof of 2.4.1. Note that if $x$ is large with respect to $s$ we have

$$
2 h^{0}(\mathscr{O}(x+2-s)) \leq h^{0}(T(x))
$$

(use (4) and the cohomology of line bundles on $S$ and $\mathbf{P}$ ). First we assume that the line bundle $J$ appearing in (2) is of the form $\mathscr{O}(y)$ for some integer $y$. By 1.1 we have $y \geq x+2-s$. Since $h^{1}(\mathbf{P}, \Omega(z))=0$ if $z \neq 0$, we see that $h^{0}(\Omega(1))=h^{0}\left(\Omega_{\mathbf{P}}(1)\right)=0$. Thus we have $y \leq x+2-s$, i.e. $y=x+2-s$. By (3) and 1.1 we conclude under the given assumption on $J$. This assumption is satisfied if $\operatorname{Pic}(S)$ is generated by $\mathscr{O}(1)$. By Noether-Lefschetz theory this is the case outside countably many proper subvarieties of the space $\mathbf{P}\left(H^{0}\left(\mathbf{P}, \mathcal{O}_{\mathbf{P}}(s)\right)\right)$ parametrizing all the surfaces of degree $s$. In particular this is true in a dense part of $\mathbf{P}\left(H^{0}\left(\mathbf{P}, \mathscr{O}_{\mathbf{P}}(s)\right)\right)$. We conclude using the next lemma.

Lemma 2.4.2. Let $\pi: \mathscr{S} \rightarrow T$ be a flat family of smooth complete surfaces (or of compact complex surfaces), and $\mathscr{E} \rightarrow \mathscr{S}$ a family (over $T$ ) of rank two vector bundles over $\mathscr{S}$. For every $t \in T$ set $S(t):=\pi^{1}(t)$ and $E_{t}: \mathscr{E} / S(t)$. Assume that $h^{0}\left(E_{t}\right)$ is the same for every $t \in T$. Then the function sending $t$ to $d\left(E_{t}\right)$, $t \in T$, is a lower semicontinuous function on $T$.

Proof. Fix $o \in T$ and set $d:=d\left(E_{o}\right)$. Fix $d$ general points $P_{1}, \ldots, P_{d}$ of $S(o)$. Taking a ramified covering of the germ $(T, o)$ we may assume that $\pi$ has $d$ disjoint sections $h_{1}, \ldots, h_{d}$, with $h_{i}(o)=P_{i}$ for every $i$; set $W(t):=$ $\left\{h_{1}(t), \ldots, h_{d}(t)\right\}$. By the semicontinuity theorem for cohomology groups we 
see that for $t$ in a small neighborhood of $o$ we have $h^{0}\left(E_{t} \otimes \mathscr{I}_{W(t), S(t)}\right) \leq$ $h^{0}\left(E_{o} \otimes \mathscr{I}_{W(o), S(o)}\right)=h^{0}\left(E_{o}\right)-2 d$. The thesis follows from the definition of $d\left(E_{t}\right)$ and the assumption that $h^{0}\left(E_{t}\right)=h^{0}\left(E_{o}\right)$ for every $t$.

(2.5) Fix $H \in \operatorname{Pic}(S), H$ ample. We want to know something about the location of singularities of a general morphism $M \rightarrow T S$ with $|n H| \subseteq\left|M^{*}\right| \subset$ $|(n+1) H|$ (meromorphic foliations with high degree polar divisor) (i.e. informations on $(s)_{0}$ for a general $s \in H^{0}\left(T S\left(M^{*}\right)\right)$. Set $d(n):=d(T S(n H))$. First fix a Lefschetz pencil (see [GM, 3.1], for a discussion in the context of foliations). Let $\gamma: S^{\prime} \rightarrow S$ be the blowing-up of the base locus $\Gamma=\{P(1), \ldots, P(d)\}$ of the pencil. By definition we have a morphism $\pi: S^{\prime} \rightarrow \mathbf{P}^{1}$ which induces a morphism $T S^{\prime} \rightarrow \pi^{*}\left(T \mathbf{P}^{1}\right)$ which induces an exact sequence similar to (1) (as in (3.2) below; this is called a Lefschetz foliation (see [GM, 3.1])). Since for every $W \subseteq\{P(1), \ldots, P(d)\}, W \neq\{P(1), \ldots, P(d)\}$, there is an integer $m$ such that for every $n \geq m T S(n H) \otimes \mathscr{I}_{W}$ is spanned by its global sections, we see that for high $n$ we have $h^{0}\left(T S(n H) \otimes \mathscr{I}_{\Gamma}\right)=h^{0}(T S(n H))-2 d$ and $T S(n H) \otimes \mathscr{I}_{\Gamma}$ is spanned by its global sections. Set $d(n, \Gamma):=\max \{t$ : for a general subset $B \subset S$ with $\operatorname{card}(B)=t-d$ we have $h^{0}\left(E(n H) \otimes \mathscr{I}_{B \cup \Gamma)}=\right.$ $\left.h^{0}(E(n H))-2 t\right\}$. The proof of 2.4.2 shows that $d(n, \Gamma) \leq d(n)$. We want to show that (under suitable assumptions) we have $d(n, \Gamma)=d(n)$. One reason for checking this equality is that, by [G3, §4], for any $L \in \operatorname{Pic}(S)$ we have $H^{0}\left(T S^{\prime} \otimes \gamma^{*}(L)\right) \cong H^{0}\left(T S \otimes L \otimes \mathscr{I}_{\Gamma}\right)$; thus the equality of $d(n, \Gamma)$ and $d(n)$ allows one to use $S^{\prime}$ (hence (1)) to have informations on $d(n)$. Warning: the equality of $d(n, \Gamma)$ and $d(n)$ is not a general vector bundle property; the fact that the rank is two seems very important and that, for fixed $\Gamma$, we may assume $E(n H)$ as positive as wanted. For a rank 3 example (with a low twist of the cotangent bundle of $\mathbf{P}^{3}$ ), see [R]; we do not know if, for fixed $\Gamma$, one can allow higher rank bundles assuming enough positivity. Since we want to prove that $d(n, \Gamma) \geq d(n)$, we may assume $2 d(n, \Gamma)<h^{0}(E(n H))$. Choose a general $B \subset S$ with $\operatorname{card}(B)=d(n, \Gamma)-d$. As in the definition of $d(E(n H))$ in (2.3.2), let ${ }_{n} J \in \operatorname{Pic}(S)$ be the saturation in $E(n H)$ of the subsheaf of $E(n H)$ spanned by $H^{0}\left(E(n H) \otimes \mathscr{I}_{B \cup \Gamma}\right)$. As in (2.3.2) we obtain an exact sequence

$$
0 \rightarrow{ }_{n} J \rightarrow E(n H) \rightarrow{ }_{n} R \otimes \mathscr{J}_{A(n)} \rightarrow 0
$$

with ${ }_{n} R \in \operatorname{Pic}(S)$ and $\operatorname{dim}(A(n))=0$.

Lemma 2.5.1. Assume $h^{0}\left(E(n H) \otimes \mathscr{I}_{\Gamma}\right)=h^{0}(E(n H))-2 d$ and $2 d(n, \Gamma) \neq$ $h^{0}(E(n H))$. Then $d(n, \Gamma)=d(n)$.

Proof. Note that by (5) and the assumption on $h^{0}\left(E(n H) \otimes \mathscr{I}_{\Gamma}\right)$, we have $h^{0}\left({ }_{n} \mathbf{J} \otimes \mathscr{I}_{\Gamma}\right)=h^{0}\left({ }_{n} \mathbf{J}\right)-d$. Moving if necessary either the set of $d(n)$ points defining the inclusion of ${ }_{n} J$ into $E(n H)$ corresponding to (5) or the Lefschetz pencil (as we prefer), we may assume $A(n) \cap \Gamma=\varnothing$. Let $V$ (resp. $V^{\prime}$ ) be the image of $H^{0}(E(n H))$ into $H^{0}\left({ }_{n} R \otimes \mathscr{J}_{A(n)}\right)$ (resp. of $H^{0}\left(E(n H) \otimes \mathscr{I}_{\Gamma}\right.$ ) into $H^{0}\left({ }_{n} R \otimes \mathscr{I}_{A(n) \cup \Gamma)}\right)$. By the assumption on $h^{0}\left(E(n H) \otimes \mathscr{I}_{\Gamma}\right) V^{\prime}$ has codimension $d$ in $V$. Hence for a general $Z \subset S$ with $\operatorname{card}(Z)=d(n)-d$ we have $h^{0}\left(E(n H) \otimes \mathscr{I}_{\text {TuZ }}\right)=h^{0}(E(n H))-2 d(n)$ (use that $\operatorname{dim}\left(V^{\prime}\right)=d(n)-d$ and $\left.h^{0}\left({ }_{n} J\right) \geq d(n)\right)$.

Remark 2.6. Assume that we work over an algebraically closed ground field with positive characteristic. Now there are problems in applying Bertini theorems. 
We do not claim that in 1.1 we may take $(s)_{0}$ reduced. Furthermore 1.2 may fail, but only for very particular linear systems; assuming one meets these linear systems, one has stronger informations concerning the problems stated in the introduction that one can hope applying 1.2. In particular the three lines after 1.3 are OK. In (2.4) the use of the Bertini theorem just before equation (4) can be avoided by using the big informations on the geometry of the sections of $\Omega_{\mathbf{P}}(2), \mathbf{P}:=\mathbf{P}^{3}$; now for the Noether-Lefschetz theorem one has to quote [DK, Exp. XIX, Theorem 2.1]. In $\S 3$ completely drop (3.5).

\section{3}

Again $S$ is a smooth complex compact surface and we use the notations introduced in $\S 1$ (in particular the notations of (1)). Fix $L \in \operatorname{Pic}(S)$; now take $T:=T S(L)$. Hence we may apply the results of the first two sections to the case of meromorphic foliations with singularities, and gain some hints on where the singularities may occur (and the dimension of the family of foliations with that assigned singularities). Essentially this section is devoted to the analysis of a few significant examples.

(3.1) Here assume $S=\mathbf{P}^{2}$ and $T \cong T \mathbf{P}^{2}(t)$ for some integer $t$. By [B, Remark 3.1], we have $d\left(T \mathbf{P}^{2}(t)\right)=\left[\left(h^{0}\left(T \mathbf{P}^{2}(t)\right)\right) / 2\right]$ for every $t \geq 1$.

(3.2) Let $g: S \rightarrow C$ be a nonconstant holomorphic map with a $C$ smooth curve. The map $g$ induces a homomorphism $T S \rightarrow g^{*}(T C)$. Call $R^{\prime}$ the image of this homomorphism and $M$ its kernel; set $R:=R^{\prime * *} . M$ and $R$ are line bundles on $S$; since $R^{\prime}$ is a rank one subsheaf of $R, R^{\prime} \cong R \otimes \mathscr{J}_{A}$ for some zero-dimensional subscheme $A$ of $S$. Thus $g$ gives us in a natural way an exact sequence (1). By definition the existence of the morphism $g$ is just the notion of holomorphic foliation with a holomorphic first integral.

(3.3) The situation described in (3.2) occurs in particular for $\mathbf{P}^{1}$-bundles, i.e. when every fiber of $g$ is smooth. The smoothness of $g$ means that $R^{\prime}=$ $g^{*}(T C)$; since $M \cong \operatorname{det}(T S) \otimes R^{*}$, we know everything. We will consider now explicitly the case $C \cong \mathbf{P}^{1}$ since in this case we will consider a more refined problem in $\S 4$. Thus assume $S \cong F_{e}, e \geq 0$ (Hirzebruch surface); $\operatorname{Pic}(S)$ has a basis $h, f$ with $f$ fiber of $g$ (hence $f^{2}=0$ ), and $h$ a minimal degree section of $g$ (hence $h^{2}=-e$ and $h \cdot f=1$ ). Now $R^{\prime}=|2 f|$ and $M=|2 h+e f|$. Hence if $e \geq 0$ and $h^{0}(T S(L)) \neq 0$, say $L=\mathscr{O}(a h+b f)$, then the sequence

$$
0 \rightarrow \mathscr{O}((a+2) h+(e+b) f) \rightarrow T S(L) \rightarrow \mathscr{O}(a h+(b+2) f) \rightarrow 0
$$

is an $h^{0}$-destabilizing sequence for $T S(L)$; hence 1.4 applies. If $e=0, T S$ splits, $T S \cong \mathscr{O}(2,0) \oplus \mathscr{O}(0,2)$ and $T S(L)$ is never $h^{0}$-stable and it is $h^{0}$ semistable if and only if $a=b$. For $e=1$ an easy calculation shows when $T S(L)$ is $h^{0}$-stable or $h^{0}$-semistable. Hence we see again (as stressed in 1.7) that in this case many problems about the locations of the singularities of meromorphic foliations are reduced to certain problems about certain line bundles. If we want simple singularities (or length-two zeroes) in general position, then 1.1 and 1.2 apply. For more complicated singularities, the method of $[\mathrm{H} 1, \mathrm{HH}$, and H2] is very powerful. The next section is made exactly to illustrate this method. For another case in which this method is applied, see $\S 6$.

(3.4) The situation described in (3.2) occurs very often: when $S$ is a nonminimal surface with Kodaira dimension $\kappa(S)=-\infty$, when $S$ has an elliptic 
fibration (even not relatively minimal) (and in particular if $\kappa(S)=1$ ), for certain classes of surfaces with $\kappa(S)=0$, for some minimal surfaces of general type; as seen in (2.4), it is a quite common situation on nonminimal surfaces (Lefschetz pencils). However usually the information obtained on $T S$ is rather weak.

(3.5) Let $S$ be a minimal surface of general type; for simplicity assume $K$ ample. By [K] TS is $K$-semistable in the sense of Mumford-Takemoto. This gives a lot of information on $T S \otimes L, L \in \operatorname{Pic}(S)$ and the possible exact sequences (1) for $T:=T S$ (see e.g. [C]); in particular this information (plus Riemann-Roch) can be used to obtain both lower bounds and upper bounds on $d(T S \otimes L)$. However, we were unable to obtain in this way nontrivial general bounds (without assuming some information on $\operatorname{Pic}(S)$ ).

This section is devoted to an exposition of (an elementary part of) the socalled Horace method ("propaganda") and an application of it to the location of singularities of meromorphic foliations on the surface $S$ (in particular when $S$ is the Hirzebruch surface $F_{1}$ ) and to the dimension of the family of meromorphic foliations with assigned singularities of a certain kind (but located as generically as possible). This application is given essentially to show that even weak versions of this method allow a user of algebraic geometry (not only the specialists of Horace method) to prove nontrivial facts and to obtain interesting examples. We stress that for the applications and the construction of examples very often it is sufficient to prove (with this method) very weak approximations of the "best possible a priori or conjectural results" that the specialists are trying to prove; these very weak approximations (and sometimes even not so weak ones) are extremely easier to prove: they can be proved by motivated nonspecialists and they require far shorter proofs. The method is due, we believe, to A. Hirschowitz (see [HH and H1]).

Let $X$ be a complete scheme (or a compact complex space), $L \in \operatorname{Pic}(X), D$ be a positive Cartier divisor on $X$ (a hypersurface). It is given a flat family of closed subschemes of $X$, say $\{W(t)\}_{t \in Y}$, parametrized by an integral variety (or an analytic disk). Note that $h^{0}\left(X, L \otimes \mathscr{I}_{W(t), X}\right)$ varies upper semicontinuously when $t$ varies in $Y$. Hence there is a Zariski open dense subset $U$ of $Y$ such that if $t \in U h^{0}\left(X, L \otimes \mathscr{I}_{W(t), X}\right)$ takes its lowest value, call it $z$, in $Y$. In favorable cases one would like to prove that $z$ is the lowest a priori possible value; one example: if $h^{i}\left(X, L \otimes \mathscr{I}_{W(t), X}\right)=0$ for every $i>1$ and every $t \in Y$, a priori the lowest possible value of $z$ is $\chi\left(X, L \otimes \mathscr{I}_{W(t), X}\right)$ and this is a case for a certain $t$ if and only if $h^{1}\left(X, L \otimes \mathscr{I}_{W(t), X}\right)=0$. We stress again that very elementary and short implementations of the Horace method are often sufficient to obtain upper bounds for the value $z$ which are sufficient for the "application": this is one of the main points for users.

Usually for the implementation of the method one uses an induction. Furthermore, by semicontinuity, to prove that $z$ takes the lowest possible value $w$ (or to prove that $z \leq w$ ) it is sufficient to find a flat extension of the family parametrized by $Y$ to a flat family parametrized by an integral $Y^{\prime}$ with $Y \subseteq Y^{\prime}$ and prove the existence of some $t^{\prime} \in Y$ with $h^{0}\left(X, L \otimes \mathscr{I}_{W(t), X}\right)=w$. One tries to find a good $W \in\{W(t)\}_{t \in Y^{\prime}}$, of the form $W=W^{\prime} \cup W^{\prime \prime}$ with $W^{\prime} \subset D$ 
and $W^{\prime \prime} \cap D=\varnothing$ (or with no irreducible component on $D$ if $\operatorname{dim}\left(W^{\prime \prime}\right)>0$ ). For such a $W$ one has the following exact sequence:

$$
0 \rightarrow L(-D) \otimes \mathscr{I}_{W^{\prime \prime}, X} \rightarrow L \otimes \mathscr{I}_{W, X} \rightarrow(L \mid D) \otimes \mathscr{I}_{W^{\prime}, D} \rightarrow 0 .
$$

Thus to show (for instance) that $h^{1}\left(X, L \otimes \mathscr{I}_{W, X}\right)=0$, it is sufficient to prove that $h^{1}\left(X, L(-D) \otimes \mathscr{I}_{W^{\prime \prime}, X}\right)=h^{1}\left(X, L \otimes \mathscr{I}_{W^{\prime}, D}\right)=0$. Thus one is reduced to an assertion on $X$ for a line bundle $L(-D)$ which is "simpler" than $L$ and to an assertion on $D$ (note that $\operatorname{dim}(D)<\operatorname{dim}(X)$ ); the second assertion is either trivial (as in the example given here in 4.1) or proved by the same method (hence with induction on $\operatorname{dim}(X)$ ); the first assertion can be either proved or reduced to a simpler assertion using again the same method applied to $L(-D)$ and a hypersurface, $D^{\prime}$. Note that for the assertion involving $L(-D)$ we may use again semicontinuity; thus it is not necessary to find a solution $W^{\prime \prime}$ with $W^{\prime \prime} \cap D=\varnothing$; in particular we may use $D$ as $D^{\prime}$ and even if $W^{\prime}$ is given and good, $W^{\prime \prime} \cup W^{\prime}$ may be not defined (in $\{W(t)\}$ ) or with bad cohomology: it does not matter. This is one of the strongest points of the Horace method. Very often one wants to take $W$ not reduced. In this case one defines $W^{\prime}$ as $W \cap D$ (scheme-theoretical intersection). It may happen that the union of $W^{\prime}$ and the closure of the part of $W$ not on $D$ is strictly smaller as scheme of $W$. In this case there is a good definition of $W^{\prime \prime}$ which makes (7) an exact sequence; define $W^{\prime \prime}$ as the residual scheme $\operatorname{Res}_{D}(W)$ of $W$ with respect to $D$; by definition the ideal sheaf of $\operatorname{Res}_{D}(W)$ in $X$ is $\operatorname{Hom}\left(\mathscr{I}_{D, X}, \mathscr{J}_{W, X}\right)$, i.e. if $g$ is a (local) equation of $D$ at $x \in X, a \in \mathscr{I}_{W^{\prime \prime}, X, x}$ if and only if $g a \in \mathscr{I}_{W, X, x}$. Here is the example of a nonreduced subscheme which will be considered on 4.1; fix $P \in S$; the first infinitesimal neighborhood of $P$ in $S$ is the lengththree 0 -dimensional subscheme $O$ of $S$ with $O_{\text {red }}=\{P\}$ and $\mathscr{I}_{O}:=\left(\mathscr{J}_{\{P\}}\right)^{2}$; we will say that $O$ is a fat point with $P$ as support. We take as surface $S$ the Hirzebruch surface $F_{1}$, i.e. the blowing-up of $\mathbf{P}^{2}$ at one point; the same method (with different constants) works for the other Hirzebruch surfaces $F_{r}$. We recall that $\operatorname{Pic}\left(F_{1}\right)$ is freely generated by the classes $h, f$ (represented by smooth rational curves) with $h^{2}=-1, h \cdot f=1, f^{2}=0$.

Proposition 4.1. Fix integers $a, b, t$ with $a \geq 5, b \geq 5, t \geq 0$. Then for $a$ general $W \subset S:=F_{1}$ with $\operatorname{card}(W)=t$, we have $\bar{h}^{0}\left(\mathscr{O}(a h+b f) \otimes \mathscr{I}_{Z}\right)=$ $h^{0}(\mathscr{O}(a h+b f))=3 t$, where $Z$ is the union of the fat points with support on the points of $W$.

We stress that if $a=b \quad 4.1$ was proved in [H2, §7].

Proof of 4.1. Taking away the fixed components of $|a h+b f|$, we may assume $b \geq a$. The proof will be divided into six steps.

Step 1. Here and until Step 5 we assume $a$ even and $b$ even. Fix a smooth $C \in|3 h+3 f|$ (hence $C$ is elliptic). We take $W=W^{\prime} \cup W^{\prime \prime}$ with $W \cap C=W^{\prime}$ and $\operatorname{card}\left(W^{\prime}\right)=3(a+b) / 2$ and let $Z$ (resp. $Z^{\prime}$ or $Z^{\prime \prime}$ ) be the union of the fat points in $W$ (resp. $W^{\prime}$ or $W^{\prime \prime}$ ). We have $\operatorname{Res}_{C}(Z)=Z^{\prime \prime} \cup W^{\prime}$ while $Z \cap C=Z^{\prime} \cap C$ is the double $Y$ of the Cartier divisor $W^{\prime}$ on $C$. Thus if $W^{\prime}$ is general enough, $h^{0}\left(C, \mathscr{O}_{C}(a h+b f) \otimes \mathscr{I}_{Y, C}\right)=h^{1}\left(C, \mathscr{O}_{C}(a h+b f) \otimes \mathscr{I}_{Y, C}\right)=$ 0 . Thus it is sufficient to prove that the union of $Z^{\prime \prime}$ and $\operatorname{card}\left(W^{\prime}\right)$ general points on $C$ imposes independent conditions to $|(a-3) h+(b-3) f|$; just to avoid introducing other notations, we will call $W^{\prime}$ the union of these (general) card $\left(W^{\prime}\right)$ points on $C$. 
Step 2. First assume $b>a$ (since we want to reduce to a statement for $|(a-3) h+(a-3) f|)$. Fix $D \in|f|$. Specialize $W^{\prime}$ in such a way that $\operatorname{card}\left(W^{\prime} \cap D\right)=2$. Now we specialize $W^{\prime \prime}$ in such a way that $\left(W^{\prime \prime} \cap D\right)=$ $(a-4) / 2$. Thus $\Gamma:=\left(W^{\prime} \cup Z^{\prime \prime}\right) \cap D$ has

$$
\left.h^{i}\left(D, \mathscr{O}_{D}((a-3) h+(b-3) f)\right) \otimes \mathscr{I}_{\Gamma, D}\right)=0 \quad \text { for } i=0,1,
$$

and we reduce to an assertion for the scheme union of $\left(W^{\prime} \backslash\left(W^{\prime} \cap D\right)\right) \cup\left(W^{\prime \prime} \cap D\right)$ and the union, $\Phi$, of $\operatorname{card}\left(W^{\prime \prime} \backslash\left(W^{\prime \prime} \cap D\right)\right)$ general fat points. We specialize $W^{\prime} \backslash\left(W^{\prime} \cap D\right)$ in such a way that it contains exactly two points of $D$; note that this specialization is not possible, but we could not put four points of $W^{\prime}$ on $D$; this is one of the powers of Horace method. Thus (again by Horace method) we are reduced to control the postulation of the union, $\mathbf{U}$, of $m$ fat points with support $U$ general on $S, 3(a+b) / 2-4$ general points on $C$ and $(a-4) / 2$ general points on $D$.

Step 3. Here we assume $b>a+1$. Set $\alpha=0$ if 4 divides $a$, and $\alpha=1$ otherwise. We specialize $U$ in such a way that $\operatorname{card}(\operatorname{supp}(U) \cap((D \backslash(D \cap C))))=$ $((a / 2)+2-\alpha) / 2$ and $U \cap C \cap D=1+\alpha$; note again that the possibility of this specialization is one of the strengths of Horace method. By Horace method we reduce to an assertion for the union of a certain number of general fat points, a certain number of general points on $C$, and a certain number of general points on $D$.

Step 4. Then we continue for $b-a-1$ times exploiting $D$ each time, until we reduce to an assertion of good postulation with respect to $|(a-3) h+(a-3) f|$ for the union of a certain number of general fat points, a certain number of general points on $C$ and a certain number of general points on $D$. However to do that we need to have enough points on $C$; if not (i.e. if $b-a$ is much larger than $a$ ) we specialize $W^{\prime \prime}$ in such a way that $W^{\prime \prime} \cap C \cap D=\varnothing$ if in the previous construction $\operatorname{card}\left(W^{\prime \prime} \cap C \cap D\right)=2$; what we need for the construction is the parity of $\operatorname{card}\left(W^{\prime \prime} \cap C \cap D\right)$. Then we take a smooth $D^{\prime} \in|h+f|$ and we continue using $D^{\prime}$ instead of $D$. At each step we specialize $W^{\prime \prime}$ in such a way that $\operatorname{card}\left(W^{\prime \prime} \cap(C \cup D) \cap D^{\prime}\right)$ is 0,1 , or 2 . At two consecutive steps the parity of $\operatorname{card}\left(W^{\prime \prime} \cap(C \cup D) \cap D^{\prime}\right)$ change and when $\operatorname{card}\left(W^{\prime \prime} \cap(C \cup D) \cap D^{\prime}\right)$ must be even, this is 0,2 , or 4 , as we prefer; hence we may use exactly the nonfat points remaining on $C \cup D$ after the first three steps (and all of them).

Step 5. Assume $a$ odd and $b$ odd. Look at Step 1. Now we take as $C$ a smooth curve in $|h+f|$ (hence irreducible and rational) and take $\operatorname{card}\left(W^{\prime}\right)=$ $(b+1) / 2$. Then we continue as in Steps 2,3 , and 4 .

Step 6. Assume $a+b$ odd. Now, with the notations of Step 1, we take as $C$ a smooth curve in $|h+2 f|$ (hence irreducible and rational) and take $\operatorname{card}\left(W^{\prime}\right)=(a+b+1) / 2$. Then we continue in the usual way.

In this short section we will make a few remarks. In (5.2) and (5.3) we will consider briefly the case of a nonalgebraic surface $S$. Recall [BPS] that for a smooth compact complex surface $S a(S)$ is the trascendence degree of the field of meromorphic functions on $S ; a(S) \in\{0,1,2\}$ and $S$ is algebraic if and only if $a(S)=2$ (and in this case $S$ is even projective).

(5.1) Note again that on any smooth surface $S$ the existence of a meromorphic foliation without singularities is equivalent to the fact that $T S$ is an 
extension of two line bundles. This seldom happens (e.g. not for smooth surface in $\mathbf{P}^{3}$ ). By definition it happens in the case considered in (3.2) when the map $g$ has nowhere vanishing differential; again this is a strong restriction but satisfied in interesting cases (e.g. hyperelliptic surfaces).

(5.2) Now assume $a(S)=1$. In this case $S$ has an elliptic fibration $g: S \rightarrow$ $C$ [BPS, VI 4.1] $g$ not necessarily relatively minimal. The discussion of (3.2) applies with the following important complement. Since every irreducible compact curve of $S$ is contained in a fiber of $g$, problems about locations of singularities handled through exact sequences like (1) (e.g. using the elliptic fibrations) can be easily reduced to assertions about linear systems and divisors on $C$.

(5.3) Now assume $a(S)=0$. In this case there is no complete description of the possible $S$, but, as we will see here, from the point of view of meromorphic foliations the situation is trivial. Set $T:=T S$. A priori two cases can occur. Either there is an exact sequence (1) for some $M, R, A$, or not. If there is no such exact sequence, there is no meromorphic foliation with singularities on $S$. Hence from now on we assume the existence of (1) (i.e. in the terminology of [EF], that $T$ is a filterable vector bundle). First note [BPS, IV 6.1(i)] that we have $h^{0}(J) \leq 1$ for every $J \in \operatorname{Pic}(S)$. Hence by (1) $h^{0}(T) \leq 2$. Since (almost by definition of the case $a(S)=0$ ) has only finitely many curves, for a general $P \in S$ and for any $J \in \operatorname{Pic}(S), h^{0}\left(T \otimes J \otimes \mathscr{J}_{\{P\}}\right)=0$, i.e. the singularities of any meromorphic foliations are bound to be on the finite curves on $S$.

(5.4) The definitions and properties studied here for singular meromorphic foliations are essentialy bimeromorphic invariant (at least for suitable polar divisors which are trivial on the exceptional curves). For singular surfaces a useful definition of meromorphic foliations not birational invariant and hence sensitive to the type of the singularities of the surface was given in [G4].

\section{6}

In this section we consider the case of singular meromorphic foliations on a complex manifold $X$ with logarithmic singularities along a normal crossing divisor $D$. First we want to describe a subbundle of $T X$ : the subbundle of vector fields with logarithmic poles along $D$ (see [De, Chapter II, §3], for the dual concept of differential forms with logarithmic poles along $D$ ). For simplicity we assume $D$ smooth. Fix a point $P \in D$; let $x_{1} \ldots, x_{n}$ be local coordinates of $X$ at $P$ such that $D=\left\{x_{1}=0\right\}$ near $P$; then at the point $P$ the sheaf $T X(-\log (D))$ is by definition the subsheaf of $T X$ generated by $\partial / \partial_{x_{i}}$ for $i \geq 2$ and by $x_{1} \partial / \partial_{x_{1}}$. We need only that $T X(-\log (D))$ is locally free and the existence of the following exact sequence:

$$
0 \rightarrow T X(-\log (D)) \rightarrow T X \rightarrow \mathscr{O}_{D}(D) \rightarrow 0 .
$$

Since $D$ is smooth the surjective map $v: T X \rightarrow \mathscr{O}_{D}(D)$ in (8) is the composition of the restriction map $T X \rightarrow T X \mid D$ and of the normal bundle surjection $T X \mid D \rightarrow \mathscr{O}_{D}(D)$. In the terminology of $[\mathrm{M}], T X(-\log (D))$ is the elementary transformation of $T X$ under the surjection $v$. We want to point out that the use of elementary transformations (again due to Hirschowitz) is one of the more powerful refinements of Horace method (see [I] (at least p. 71) and [B1]). Fix $M \in \operatorname{Pic}(X)$; a singular meromorphic foliation by curves with logarithmic poles along $D$ is a nonzero morphism $M \rightarrow T X(-\log (D))$; it seems a nice concept. 
The aim of this section is to show that general vector bundle techniques are useful even in this case (we will apply the Horace method). We will consider the case of $X:=S:=\mathbf{P}^{2}$. Here is the result.

Theorem 6.1. Fix an integer $s \geq 1$. There is an integer a(s) with the following property. Fix integers $t$ and $c$ with $t \geq a(s)$ and $c \geq a(s)$. Then for a general $D \in|\mathscr{O}(s)|$ and a general $W \subset \mathbf{P}^{2}$ with $\operatorname{card}(W)=c$, we have

$$
h^{0}\left(T \mathbf{P}^{2}(-\log (D))(t) \otimes \mathscr{I}_{W}\right) \leq \max \left(0, h^{0}\left(T \mathbf{P}^{2}(-\log (D))(t)\right)-2 c+a(s)\right) .
$$

Note that in Theorem 6.1 the integer $a(s)$ does not depend on $t$ and $c$; hence 6.1 claims that for $T S(-\log (D)) \quad W$ gives at least almost the maximal a priori possible number of conditions to $H^{0}\left(T \mathbf{P}^{2}(-\log (D))(t)\right.$ ) (which is $\min \left(2 c, h^{0}\left(T P^{2}(-\log (D))(t)\right)\right)$.

Proof of 6.1. Fix a smooth conic $C \subset S:=P^{2}$. It is very well known (see for instance $[\mathrm{B}, 1.3])$ that $T S \mid C$ is the direct sum of two line bundles of degree 3. Fix $D \in|\mathscr{O}(s)|, D$ smooth and intersecting $C$ transversally at $2 s$ points. There is an integer $b(s)$ such that for every $k \geq b(s)$ we have $h^{i}(T S(-\log (D))(k))=0$ for $i=1,2$. We note that this integer $s$ depends only on $s$, not on the choice of $D$; this follows from general principles (semicontinuity and the fact that the set of plane curves of degree $s$ forms an algebraic family), but using (8) one can find an explicit $a(s)$. In particular we note that by $(8)$ and the definition of $b(s)$ for $k \geq b(s)$ the integer $h^{0}(T S(-\log (D))(k+2))-h^{0}(T S(-\log (D))(k))$ is even. Consider the restriction of $(8)$ to $C$,

$$
0 \rightarrow(T S(-\log (D)))|C \rightarrow(T S)| C \rightarrow \mathscr{O}_{C \cap D} \rightarrow 0 .
$$

By (9) $(T S(-\log (D))) \mid C$ has degree $6-2 s$. Consider the following assumption (*).

Assumption $(*)$. Assume that $(T S(-\log (D))) \mid C$ is the direct sum of two line bundles of degree $3-s$.

Assume $(*)$. As in [B, 3.1], we can use the Horace method using $C$ and the assumption $(*)$ to obtain 6.1 for the integer $k+2$ (with $k \geq b(s)$ ) if we have 6.1 for the integer $k$. Thus, assuming $(*)$, we obtain 6.1 taking for instance $a(s):=h^{0}(T S(-\log (D))(b(s)+1))$ (which is huge). We have to justify $(*)$. It is at this point that we need the generality of $D$. Fix any $D$ intersecting $C$ transversally. We will prove $(*)$ not for $D$ but for any smooth $D^{\prime}$ with $D^{\prime} \cap C=D \cap C$ and such that the tangent lines to $D^{\prime}$ at the points of $D \cap C$ are general enough (hence varying $D$ and $D \cap C$ for general $D^{\prime}$ ). By the definition of the surjection $\tau$ in (9) (restriction to $C$ of the composition of a restriction map and a normal bundle sequence of $D$ ) at $x \in C \cap D$ the map $\tau(x)$ of the fibers has the line $T_{x} D$ as kernel. Thus, by semicontinuity, to prove that the general such $D^{\prime}$ satisfies $(*)$ it is sufficient to check the following fact: given a balanced rank two vector bundle $E$ on a smooth rational curve, $C$, and an even subset $W \subset C$ and a sheaf $G$ on $C$ with support on $W$ and one-dimensional stalk at each point, the general surjection $E \rightarrow G$ has as kernel a balanced rank two vector bundle on $C$. This assertion is well known; furthermore, by semicontinuity, it can be proved in the following way; write $W=U \cup V$ with $\operatorname{card}(U)=\operatorname{card}(V)=\operatorname{card}(W) / 2$; map one of the two factors of $E \mid W$ surjectively onto $G \mid U$ and to zero on $G \mid V$ and do the opposite for the other factor of $E$. 
A lower bound for the integer $a(s)$ which arises easily from the proof of 6.1 is very bad; to obtain more, one can take instead of an integer $a(s)$ two integers $a^{\prime}(s)$ and $a^{\prime \prime}(s)$ and require $t \geq a^{\prime}(s)$ and $c \geq a^{\prime \prime}(s)$.

Remark 6.2. Assume that $D \subset S:=\mathbf{P}^{2}$ is a line. Then $T S(-\log (D)) \cong \mathscr{O}_{S}(1) \oplus$ $\mathscr{O}_{S}(1)$.

Proof. Since $T S \mid D \cong \mathscr{O}_{D}(2) \oplus \mathscr{O}_{D}(1)$, by (8) we may apply [B1, Lemma 1.2].

Proposition 6.3. Let $D \subset \mathbf{P}^{2}$ be a smooth curve. Then $\mathbf{P}^{2}$ has a nonsingular meromorphic foliation with logarithmic poles along $D$ if and only if $D$ is a line.

Proof. The "if part" follows immediately from 6.2.

"Only if part": Set $s:=\operatorname{deg}(D)$. Since $h^{1}(\mathscr{O}(t))=0$ for every $t$, if $T S(-\log (D))(k)$ is an extension of two line bundles (which, as in (5.1) means "nonsingular" for the foliation), then it is a direct sum of two line bundles; hence it has trivial cohomology. Since $\mathscr{O}_{D}(D) \cong \mathscr{O}_{D}(s)$, by $(8)$ we get $h^{0}\left(T \mathbf{P}^{2}(-s)\right) \neq$ 0 , hence $s \leq 1$.

By (8) we can calculate the Chern classes of $T X(-\log (D)$ ) (hence obtain a strong tool to prove that, on certain $X$, there is no meromorphic foliation nonsingular except for logarithmic singularities). As shown in 6.3 and in the proof of 6.1, to obtain results sharper than 6.1 (or on other surfaces) it is very important to have more detailed informations on $T S(-\log (D))$. We can describe $T S(-\log (D))$ for other pairs $(S, D)$.

(6.4) Assume $S=\mathbf{P}^{2}, D$ a smooth conic. We want to check that

$$
T S(-\log (D)) \cong T S(-1) \text {. }
$$

By (3.1) this will give that in 6.1 we may take $a(2)=1$ and $b(2)=0$. Set $E:=T S(-\log (D))(-1)$. By $(8)$ we have $h^{0}(E(-1))=0, c_{1}(E)=-1$ and $c_{2}(E)=1$. We claim that $h^{0}(E)=0$. Assume the claim (i.e. almost by definition of stability on $\mathbf{P}^{2}$ that $E$ is stable). Then $E \cong T S(-2)$ by [Hu, 8.1]. Assume $h^{0}(E) \neq 0$. Then there is an integer $k>0$ (indeed $k=1$ ), such that $H^{0}(E(-k))$ has a section $s$ with $\operatorname{dim}\left((s)_{0}\right)=0$. Hence $s$ induces an exact sequence like (1) with $T=E, M=\mathscr{O}(k)$ and $R=\mathscr{O}(-k-1)$. Thus for a general line $J, E \mid J \cong \mathscr{O}_{J}(k) \oplus \mathscr{O}_{J}(-k-1)$. Take as $J$ a line not tangent to $D$. Since the surjection in (8) is induced by the normal bundle sequence of $D$, one checks that $T S(-\log (D)) \mid J \cong \mathscr{O}_{J} \oplus \mathscr{O}_{J}(-1)$, contradiction.

(6.5) As shown in the previous proofs, much information on $T S(-\log (D))$ is gained restricting this bundle to suitable curves. In turn by the definition of the surjection in (8), the restricted bundle depends on how the intersection of the chosen curve with $D$ (at all points!) is, the easiest case being when the two curves are transversal. Consider for instance the case $S=F_{e}, e \geq 0$, and $D \in|h+x f|$ for some $x \geq e, D$ smooth (hence rational). Let $\pi: S \rightarrow \mathbf{P}^{1}$ be the fibration with $D$ as a section. Let $J$ be a fiber of $\pi$, i.e. $J \in|f|$. Since $J \cdot D=1$, by the adjunction formula and the definition of the maps in (8) we see that $T S(-\log (D)) \mid J$ has splitting type $(1,0)$ for every $J$. Thus by the base change theorem [OSS] one checks that $\left(\pi^{*}\left(\pi_{*}(T S(-\log (D))(-h))\right)\right)(h)$ is a rank one subbundle of $T S(-\log (D))$, i.e. $T S(-\log (D))$ is the extension of two line bundles, i.e. $S$ has a meromorphic foliation with only logarithmic singularities on $D$. 
A warning on the terminology used: a nonsingular meromomorphic foliation (i.e. a foliation corresponding to an exact sequence (1) with $A=\varnothing$ ) is not necessarily a holomorphic (everywhere nonsingular) foliation (in the classical sense) (which is the case $M=\mathscr{O}_{S}$ and $A=\varnothing$ ); the same is true for foliations with logarithmic poles.

\section{REFERENCES}

[B] E. Ballico, On the homogeneous ideal of $s$ general points in $\mathbf{P}^{3}$, J. Algebra 106 (1987), 46-52.

[B1] , On the homogeneous ideal of projectively normal curves, Ann. Mat. Pura Appl. (4) 154 (1989), 83-90.

[BPS] W. Barth, C. Peters and A. Van de Ven, Compact complex surfaces, Ergebnisse der Math. Bd. 4, Springer-Verlag, 1984.

[C] A. Corti, Polynomial bounds for the number of automorphisms of a surface of general type, Ann. Sci. École Norm. Sup. (4) 24 (1991), 113-137.

[De] P. Deligne, Equations différentielles a points singuliers réguliers, Lecture Notes in Math., vol. 163, Springer-Verlag, 1970.

[DK] P. Deligne and N. Katz, Groupes de monodromie en géométrie algébriques. II, (SGA 7 II), Lecture Notes in Math., vol. 340, Springer-Verlag, 1973.

[Ea] A. Eastwood, Collision de biais et application à l'interpolation, Manuscripta Math. 67 (1990), 227-249.

[EF] G. Elencwajg and O. Forster, Vector bundles on manifolds without divisors and a theorem on deformations, Ann. Inst. Fourier 32 (1982), 25-51.

[G1] X. Gomez-Mont, The transverse dynamics of a holomorphic flow, Ann. of Math. (2) 127 (1988), 49-92.

[G2] $\_$, Holomorphic foliations in ruled surfaces, Trans. Amer. Math. Soc. 312 (1989), 179201.

[G3] _ On closed leaves of holomorphic foliations by curves, Algebraic Geometry and Complex Analysis (Patzcuaro, 1987), Lecture Notes in Math., vol. 1414, Springer-Verlag, 1989, pp. 61-98.

[G4] - Foliations by curves of complex analytic spaces, Contemporary Math., vol. 58, Amer. Math. Soc., Providence, RI, 1987, pp. 123-141.

[G5] _ Integrals for holomorphic foliations with singularities having all leaves compact, Ann. Inst. Fourier (Grenoble) 39 (1989), 451-458.

[GK] X. Gomez-Mont and G. Kempf, Stability of meromorphic vector fields in projective spaces, Comment. Math. Helv. 64 (1989), 462-473.

[GM] X. Gomez-Mont and J. Mucino, Persistent cycles for holomorphic foliations having a meromorphic first integral, Holomorphic Dynamics, (Proceedings Mexico 1986), Lecture Notes in Math., vol. 1345, Springer-Verlag, 1989.

[HH] R. Hartshorne and A. Hirschowitz, Droites en position générale dans l'espace projectif, Algebraic Grometry, (Proc. La Rabida 1981), Lecture Notes in Math., vol. 961, Springer-Verlag, 1982, pp. 169-189.

[H1] A. Hirschowitz, Sur la postulation générique des courbes rationelles, Acta Math. 146 (1981), 209-230.

[H2] __ La methode d' Horace pour l'interpolation à plusieurs variables, Manuscripta Math. 50 (1985), 337-388.

[Hu] K. Hulek, Stable rank 2 vector bundles on $\mathbf{P}_{2}$ with $c_{1}$ odd, Math. Ann. 242 (1979), 241-266.

[K] S. Kobayashi, Differential geometry of complex vector bundles, Publ. Math. Soc. Japan 15 (1987).

[I] M. Idà, On the homogeneous ideal of the generic union of lines in $\mathbf{P}^{3}, \mathbf{J}$. Reine Angew Math. 403 (1990), 67-153. 
[M] M. Maruyama, Elementary transformations of algebraic vector bundles, Algebraic Geometry, (Proc. La Rabida 1981), Lecture Notes in Math., vol. 961, Springer-Verlag, 1982, pp. 241-266.

[OSS] C. Okonek, M. Schneider and H. Spindler, Vector bundles on complex projective spaces, Progress in Math., vol. 3, Birkhäuser, 1980.

[Pe] D. Perrin, Courbes passant par $m$ points généraux de $\mathrm{P}^{3}$, Mém. Soc. Math. France 28/29 (1987).

[R] L. Roberts, The ideal generation conjecture for 28 points in $\mathbf{P}^{3}$, Canad. J. Math. 38 (1986), 1228-1238; Erratum to "The ideal generation conjecture for 28 points in $\mathbf{P}^{3}$ ", Canad. J. Math. 39 (1987), 512.

Department of Mathematics, University of Trento, 38050 Povo (TN), Italy

E-mail address: (bitnet) ballico@itncisca; (decnet) itnvax::ballico 